AIAA 2002-3761

\title{
THE IRIS-GUS SHUTTLE BORNE UPPER STAGE SYSTEM
}

\author{
Craig Tooley \\ NAS A Goddard Space Flight Center, Greenbelt, MD \\ Martin Houghton \\ NASA Goddard Space Flight Center, Greenbelt, MD \\ Luigi Bussolino \\ Alenia Aerospazio, Turin Italy \\ Paul Connors \\ Swales Aerospace, Beltsville, MD
}

\begin{abstract}
This paper describes the IRIS-GUS upper stage system that will be used to launch NASA's Triana Observatory from the Space Shuttle. Triana is a pathfinder earth science mission being executed on a rapid schedule and small budget, therefore the mission's upper stage solution had to be a system that could be fielded quickly at relatively low cost and risk. The building of the IRIS-GUS system was necessary because NASA lost the capability to launch moderately sized upper stage missions from the Space Shuttle when the PAM-D system was retired. The IRIS-GUS system restores this capability. The resulting system is a hybrid which mates the existing, flight proven IRIS (Italian Research Interim Stage) airborne support equipment to a new upper stage, the Gyroscopic Upper Stage (GUS) built by the GSFC for Triana. Although a new system, the GUS exploits flight proven hardware and design approaches in most subsystems, in some cases implementing proven design approaches with state-of-the-art electronics. This paper describes the IRIS-GUS upper stage system elements, performance capabilities, and payload interfaces.
\end{abstract}

\section{Background}

NASA's Triana mission will place a small earth observing spacecraft at the earth-sun L1 libration point in order to perform earth science and return high definition continuous images of the entire sunlit side of the planet. Triana is a pathfinder mission in that it is the first earth science spacecraft to provide a continuous synoptic view of the planet ${ }^{(1)}$. The mission was baselined to be a relatively low cost, quick reaction mission that leverages existing assets to achieve its goals.

Copyrighı $\odot$ American Institule of Acronautics and Astronautics, Inc. No copyright is assented in the United States under Title 17. U.S. Code. The L.S. Guvernment has assered in the United Stales ull rights under the copyright claim herein for royaly-free license to exere all other rights are reserved by the copyright owner
Part of that baseline was that Triana was to be launched as a Space Shuttle secondary payload.

To reach L1 Triana must employ an upper stage to boost it from the Shuttle parking orbit into the L1 transit trajectory ${ }^{(2)}$. The search for appropriately sized shuttle upper stage systems and supporting Airborne Support Equipment (ASE) quickly lead to the realization that the US capability in this area, namely the PAM-D system, had been retired from service many years prior and was not retrievable. Fortunately the Italian Space Agency had also built and fielded a similar system, the IRIS, and maintained the ASE in clean storage in anticipation of future use. The IRIS system was originally comprised of the spin Table-launcher ASE, which remains in the Shuttle bay and a unique solid motor upper stage, designed to take moderately sized satellites to geosynchronous transfer orbit. The IRIS upper stage was no longer available.

To meet the mission launch requirements the Triana project at the NASA Goddard Space Flight Center designed and built a new upper stage, the Gyroscopic Upper Stage (GUS), to be used in conjunction with the IRIS ASE. The combination of the IRIS ASE and the GUS restore the capability to launch moderately sized satellites into higher energy trajectories from the Space Shuttle. The complete system is shown in Figure 1 including the Triana Observatory and is shown in a typical Orbiter payload bay manifest in Figure 2 .

\section{Mission Scenario}

To understand the function and purpose of the systems that make up IRIS-GUS it is necessary to understand the execution of a typical satellite deployment mission. In Table 1 a simplified chronological outline of a mission is given. 


\begin{tabular}{|l|l|}
\hline \multicolumn{2}{|c|}{ Table - 1 Typical IRIS-GUS Space Shuttle Satellite Deployment Mission } \\
\hline \multicolumn{1}{|c|}{ Flight Event } & \multicolumn{1}{c|}{ Execution Time } \\
\hline Space Shuttle Launch & Begins Flight Day 1 \\
\hline IRIS ASE powered up & Within 30 minutes of Payload Bay Door opening \\
\hline IRIS ASE functional checkout executed (1 hour) & Flight Day 1 \\
\hline Functional Check-out of Satellite (4 hours) & Flight Day 2 \\
\hline Deployment Sequence (20 minutes) & Flight Day 2 to 8 depending on Shuttle mission \\
\hline Orbiter maneuvers to deploy attitude & Within deployment sequence \\
\hline Spin up (45-60 rpm) & Spin rate depends on satellite requirements \\
\hline Deployment from Orbiter & Exact deployment time per satellite requirements \\
\hline Coast phase & Deploy +0:00 to Deploy $+47: 00$ (min: sec) \\
\hline Orbiter maneuvers to safe distance and attitude & Within coast phase \\
\hline Solid Rocket Motor ignition & Deploy $+47: 00$ \\
\hline Motor Burnout & Deploy $+48: 28$ (Star-48B) \\
\hline Coast to deplete residual thrust & Deploy $+48: 28$ to Deploy $+52: 30$ \\
\hline Satellite separation from GUS & Deploy $+52: 30$ \\
\hline
\end{tabular}

\section{System Description}

\section{IRIS ASE}

The IRIS ASE consists of a motorized spin Table equipped with a spring driven launcher, control and support avionics, and the structural support /thermal protection subsystems ${ }^{(3)}$. The composite spacecraft (GUS plus satellite) is attached to the spin Table via pyrotechnically actuated clamp-band system. Two retractable lateral supports that attach to the GUS, the Grabbers, provide additional support during launch and landing. The IRIS spring driven ejection system deploys the spinning composite spacecraft with a velocity of approximately $1 \mathrm{~m} / \mathrm{s}$ relative to the Space Shuttle Orbiter and a spin rate of 45 to 60 $\mathrm{rpm}$. The Orbiter establishes the deployment inertial pointing.

The IRIS ASE structure is a space-frame design attached to the Orbiter with a standard five-trunnion interface. IRIS is covered with thermal insulation and equipped with heater panels and a retractable sunshield, which together provide a benign on-orbit thermal environment for payloads prior to deployment. The IRIS ASE avionics primarily provide control and monitoring of the IRIS and GUS systems but can be utilized to provide limited services to the satellite. The IRIS ASE is depicted in Figure 3. The IRIS ASE occupies a quarter of the Space Shuttle Orbiter payload bay and weighs 1083 $\mathrm{kg}$ without the GUS and satellite. The IRIS was flown in 1992 and successfully deployed the LAGEOS II satellite. Additional details about the design of the IRIS ASE can be found in the references ${ }^{(3)}$.

\section{Gyroscopic Upper Stage}

The GUS is a new spin stabilized upper stage designed to adapt the Star-48B solid rocket motor to the IRIS ASE and provide the command, control and stabilization during upper stage flight necessary to meet the Triana mission's trajectory requirements. The GUS vehicle and a GUS system block diagram are shown in Figures 4 and 5. The GUS is designed to be two fault tolerant to failures leading to catastrophic hazards until safely separated from the Orbiter and one fault tolerant against mission success failures. GUS subsystems and their functions are briefly described below:

\section{Solid Rocket Motor}

The Thiokol Star- $48 \mathrm{~B}^{(4)}$ was selected for the GUS. The Star- $48 \mathrm{~B}$ is flight proven and was previously flown on the Shuttle as part of the PAM-D upper stage system. GUS uses the Shuttle qualified Thiokol Safe and Arm model 2134B and the associated ignition ordnance. As will be discussed later GUS can be fitted with smaller Star motors should that be desirable.

\section{Sequencer System}

The functional heart of the GUS is the Triana Event Sequencer Subsystem (TESS), which is comprised 
of two identical redundant sequencer units. The sequencers use Field Programmable Gate Array (FPGA) based timers and electromechanical relays to execute the pre-programmed GUS flight functional timeline.

\section{Power System}

Two Ag-Zn 13 amp-hour 28 volt batteries provide redundant power to GUS systems and can supply limited power to the attached satellite during the upper stage free-flight coast period prior to rocket motor ignition. Power is switched on by the Power Contactor Unit, which is controlled by microswitches that sense separation from the IRIS spin Table at deployment.

\section{Nutation Control System}

In order to prevent performance degradation due to nutation of the spinning composite spacecraft the GUS has a fully redundant active Nutation Control System (NCS). The NCS is microprocessor controlled and employs angular rate sensors and cold gas $\left(\mathrm{GN}_{2}\right)$ thrusters. The system has two control modes, a coast mode designed to counteract nutation caused principally by initial deployment disturbances and satellite fuel slosh, and a powered flight mode designed to control nutation growth induced by disturbance phenomena associated with the Star-48B motor.

\section{Telemetry System}

A single string S-band telemetry system provides GUS engineering telemetry to the ground during the coast and powered flight phases of the mission. GUS operates autonomously once deployed and has no RF command capability.

\section{Mechanical Systems}

The GUS structure is an all-metal aluminum design and accommodates the GUS subsystems in addition to interfacing with both the IRIS and the satellite. The configuration was dictated by the requirement to adapt the Star-48B to the IRIS ASE. The mechanical systems include the satellite interface, which is a clamp-band system identical to the Delta II 3712C Payload Attach Fitting.

\section{Thermal Control}

GUS thermal control is achieved using a conventional combination of passive thermal insulation and surface coatings and a limited number of active, thermostatically controlled resistive heaters. In addition to maintaining GUS systems within limits during all mission phases the thermal control system protects the satellite from excessive heating during the powered flight and motor thermal soak-back period after burnout.

\section{System Performance}

The IRIS-GUS upper stage system performance was characterized in detail for the Triana mission. Those verified capabilities are summarized in the Table 2. Aspects of the satellite configuration such as mass properties and energy dissipation sources affect performance and thus improved performance is possible, depending on the specific mission under consideration. Satellite Accommodation

The physical accommodation of a satellite payload on the IRIS-GUS system is summarized below. Detailed design information is available and is being consolidated in an IRIS-GUS User Guide.

\section{Mechanical}

The available volume for the satellite payload is shown in Figure 6. The satellite interface to GUS is a $958.85 \mathrm{~mm}$ clamp band attach fitting identical to the Delta II 3712C payload interface. Satellites being launched by IRIS-GUS must be capable of being spin balanced. The structural loading environment is commensurate with other medium sized Shuttle payloads and NASA GSFC can supply preliminary design load factors appropriate for potential IRIS-GUS satellites.

\section{Thermal}

Prior to deployment from the IRIS ASE a benign thermal environment is provided by the IRIS ASE thermal control system. Typically the range of 5 to $30^{\circ} \mathrm{C}$ will bound the temperature extremes for a quiescent satellite awaiting deployment. Once deployed the satellite must relay on its own thermal control system during the upper stage flight phase. The GUS is designed to limit the maximum temperature at the satellite attachment interface to 50 ${ }^{\circ} \mathrm{C}$. The peak temperature occurs during the motor soak-back period prior to separation.

\section{Electrical}

Two 60-pin connectors span the GUS-satellite interface and are primarily used to route power, commands, and telemetry from the satellite to the 
Space Shuttle and ground controllers during operations prior to deployment. The IRIS ASE has the capability to provide for a limited number of power lines, discrete commands, and both analog and bi-level monitors. IRIS can also control a second Safe and Arm, usually required if the satellite carries an Apogee Kick Motor. More complex satellites may require direct interfaces to Orbiter systems for power, serial command and telemetry and safety monitoring of hazardous systems.

The GUS-to-satellite interface connectors can also be used to bring limited power from the GUS batteries to the satellite during the coast period and provide break-wires for signaling separation from GUS to the satellite.

The Triana mission utilizes a suite of auxiliary electronics drawn from the Hubble Space Telescope Space Shuttle servicing missions to interface with the Orbiter systems to provide serial commanding and telemetry as well as power, including battery charging. An attractive option of intermediate complexity is simply augmenting the basic IRIS ASE capabilities with a direct serial interface from the satellite to the crew cabin payload laptop computer.

\section{Mission Integration}

The IRIS-GUS system is designed to be compatible with the standard Space Shuttle integration flow for secondary payloads. Typically the Space Shuttle mission integration planning process begins about 22 months prior to the launch with actual delivery of the satellite to the launch site occurring about four months prior to launch. Mission specific IRIS-GUS to satellite engineering and interface testing is also accomplished within this timeframe. More information about the details of the Space Shuttle payload integration process can be found in the references ${ }^{(5)}$.

\section{Status of IRIS-GUS}

The IRIS-GUS system has been built and functionally tested in support of the Triana mission. Currently the Triana mission is awaiting establishment of a Shuttle launch date. The mission was originally manifested to launch in 2001 but Shuttle program manifest considerations resulted in a delay. When NASA establishes a launch date the environmental testing of the GUS will be completed and the IRIS ASE will undergo an acceptance thermal vacuum test. This will bring the IRIS-GUS system to the point of full flight readiness.

\section{References}

1) "The Triana Mission Implementation- A Unique Mission with a Unique Approach", J. Watzin, 2001

2) "Triana Mission Design", M. Beckman and J.J. Guzman, 1999,AAS 99-400

3) IRIS System and Future Application to Expendable Launchers, L. Bussolino, F. Varesio, G. Rum, and C. Portelli, 7 Feb. 1991

4) Thiokol Corporation Space Motor Catalog, 1991 Ed., Alliant Techsystems, Inc.

5) Web Page: shuttlepayloads.jsc.nasa.gov 


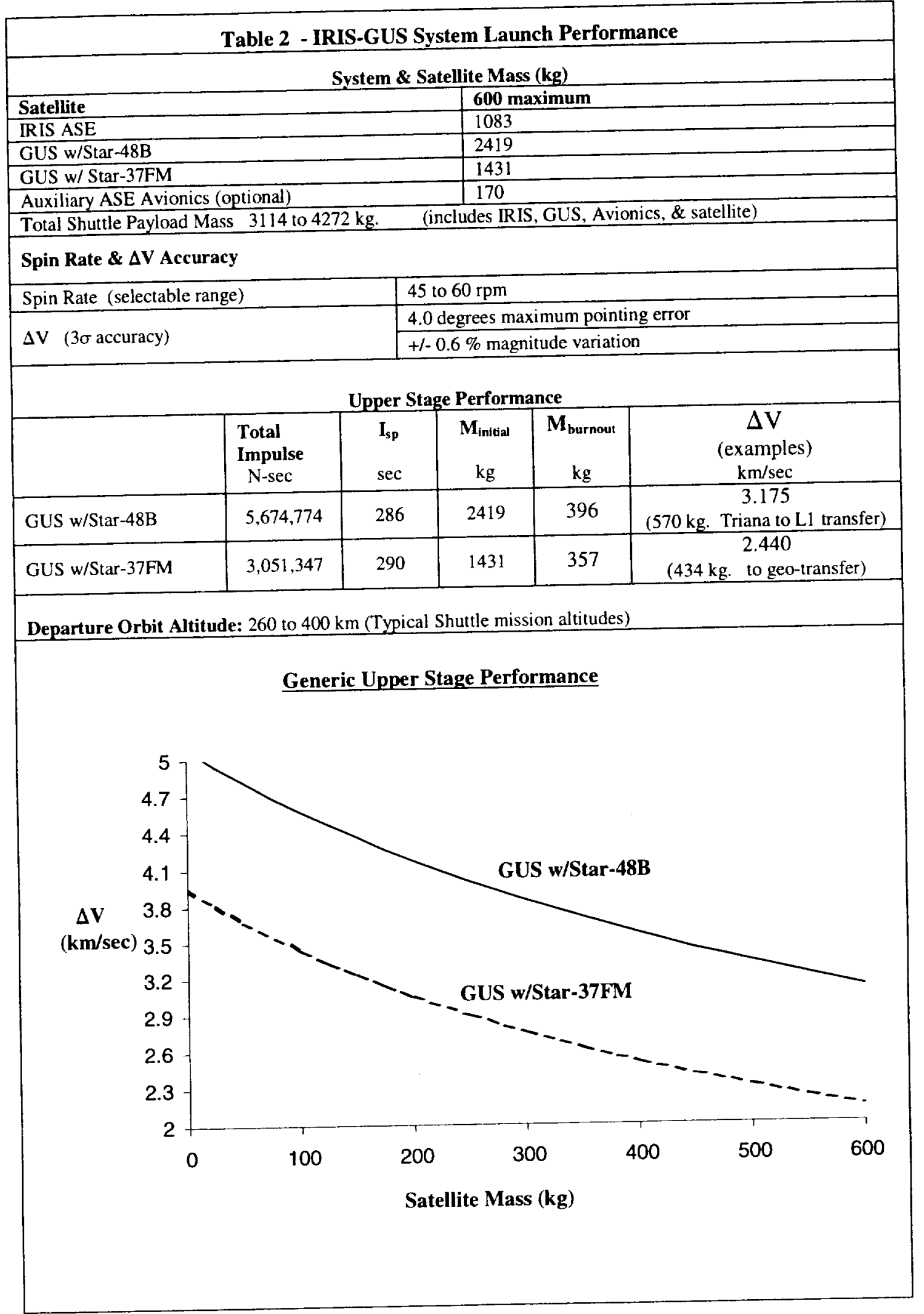




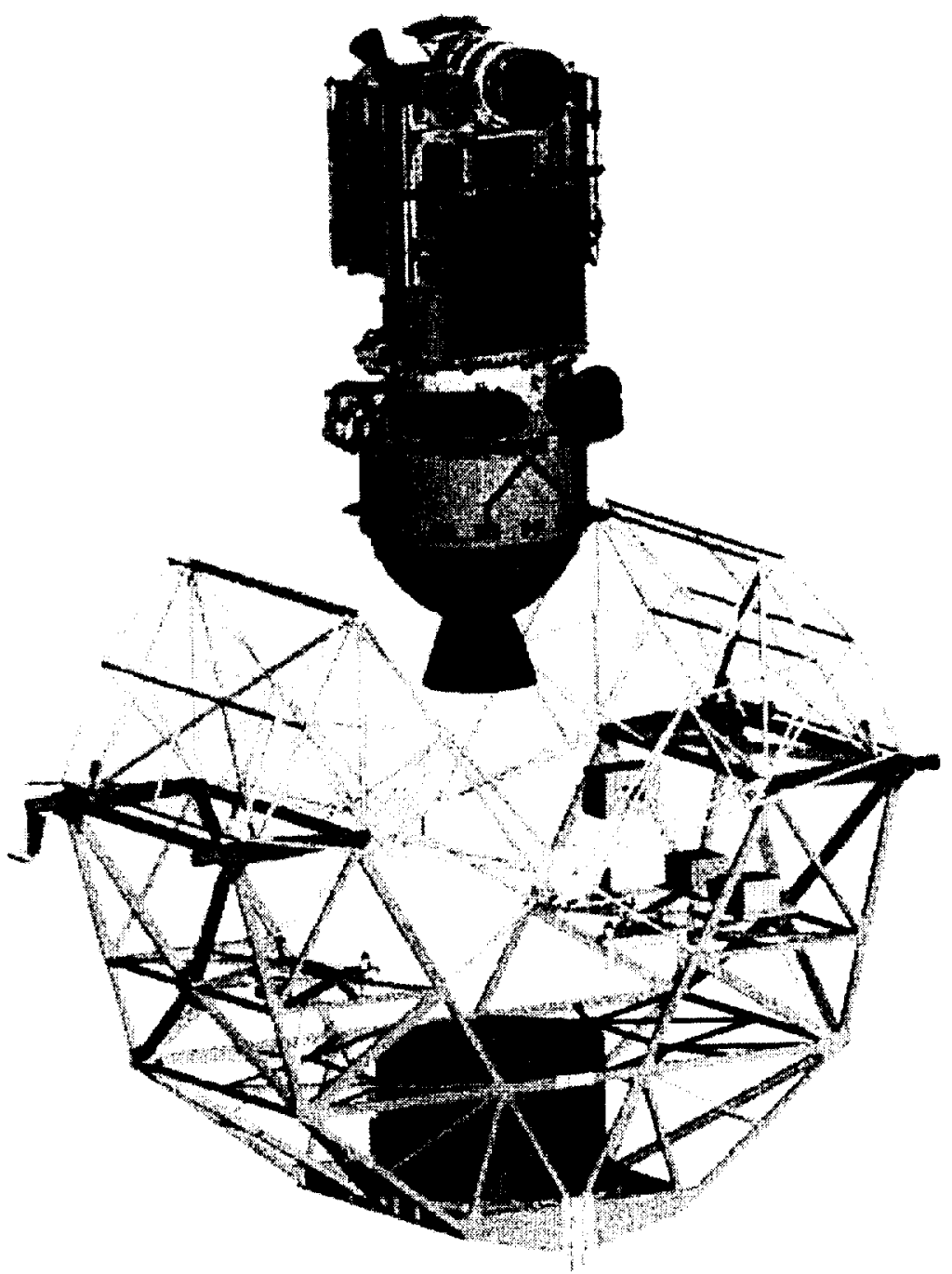

Figure 1 IRIS-GUS System shown with Triana Observatory 


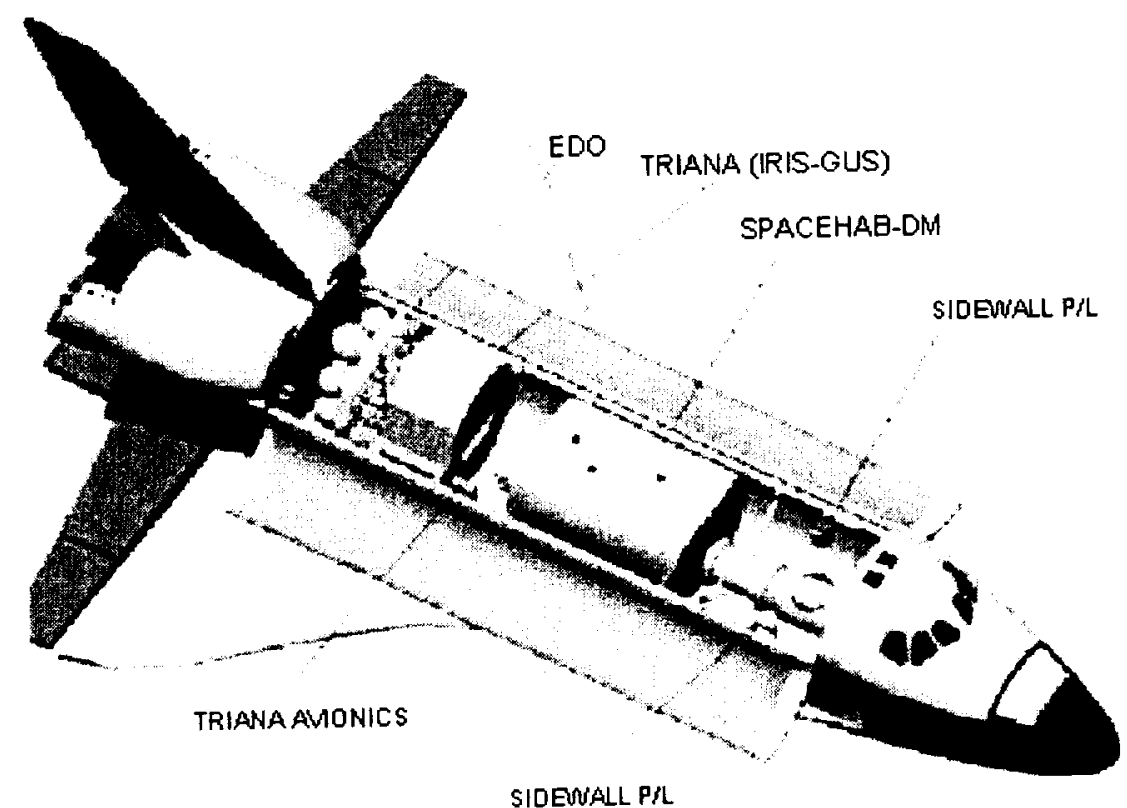

Figure 2 IRIS-GUS System Manifested on a Typical Space Shuttle Research Mission

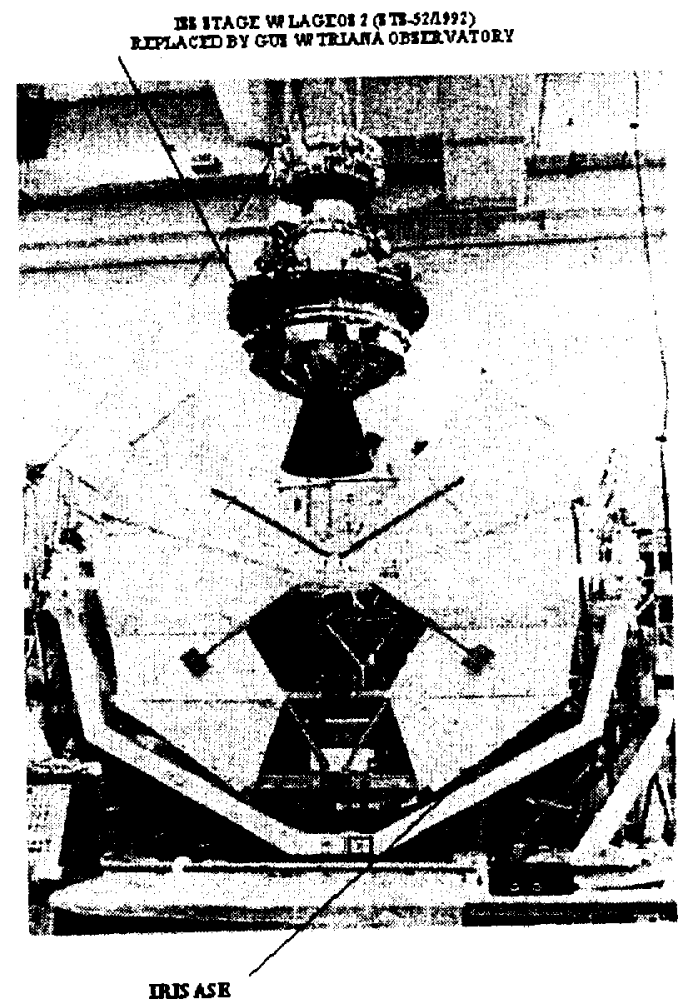

Figure 3 IRIS ASE with Original Solid Rocket Stage and the LAGEOS II Satellite 


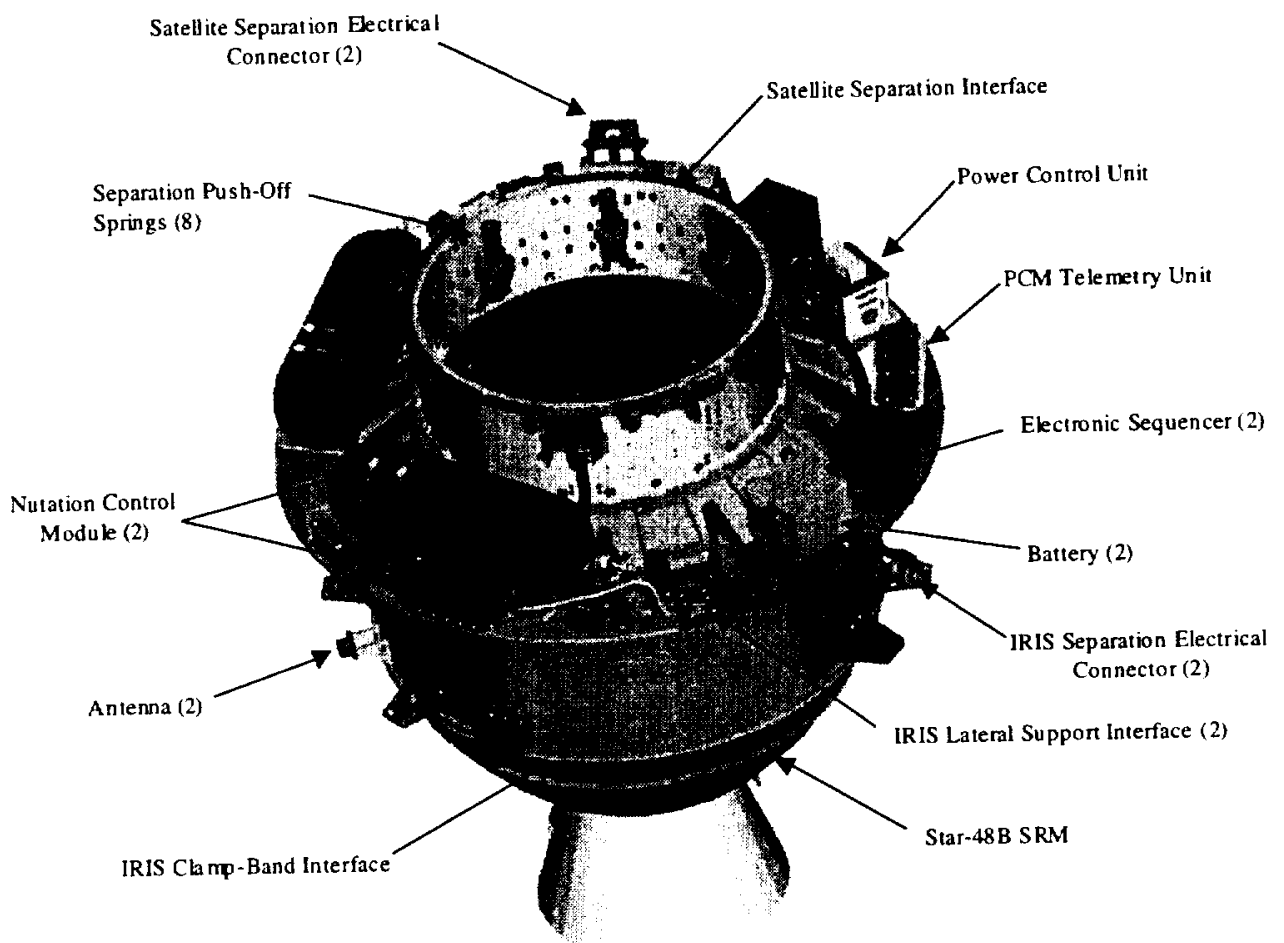

Figure 4 Gyroscopic Upper Stage (GUS)

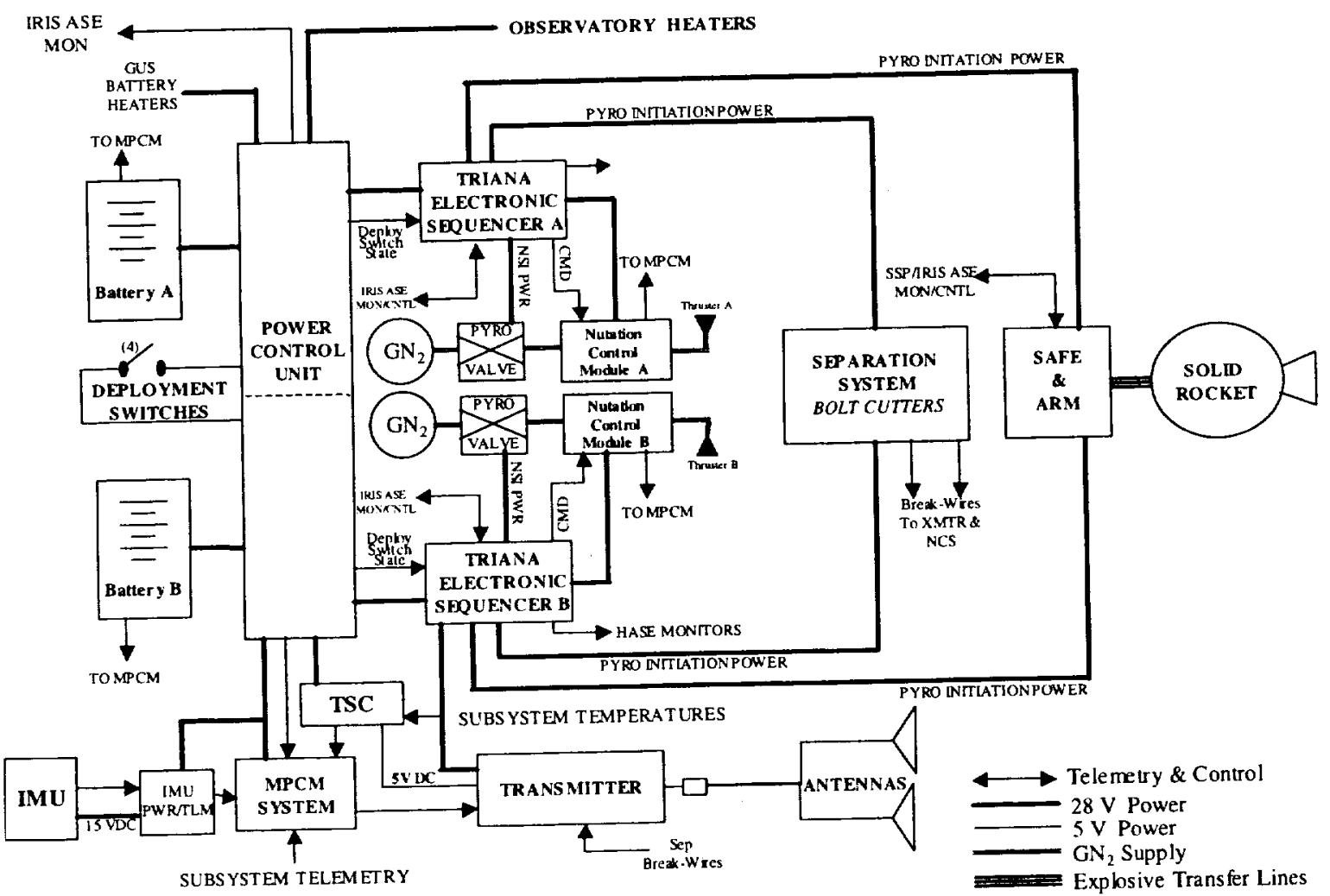

Figure 5 GUS System Block Diagram 


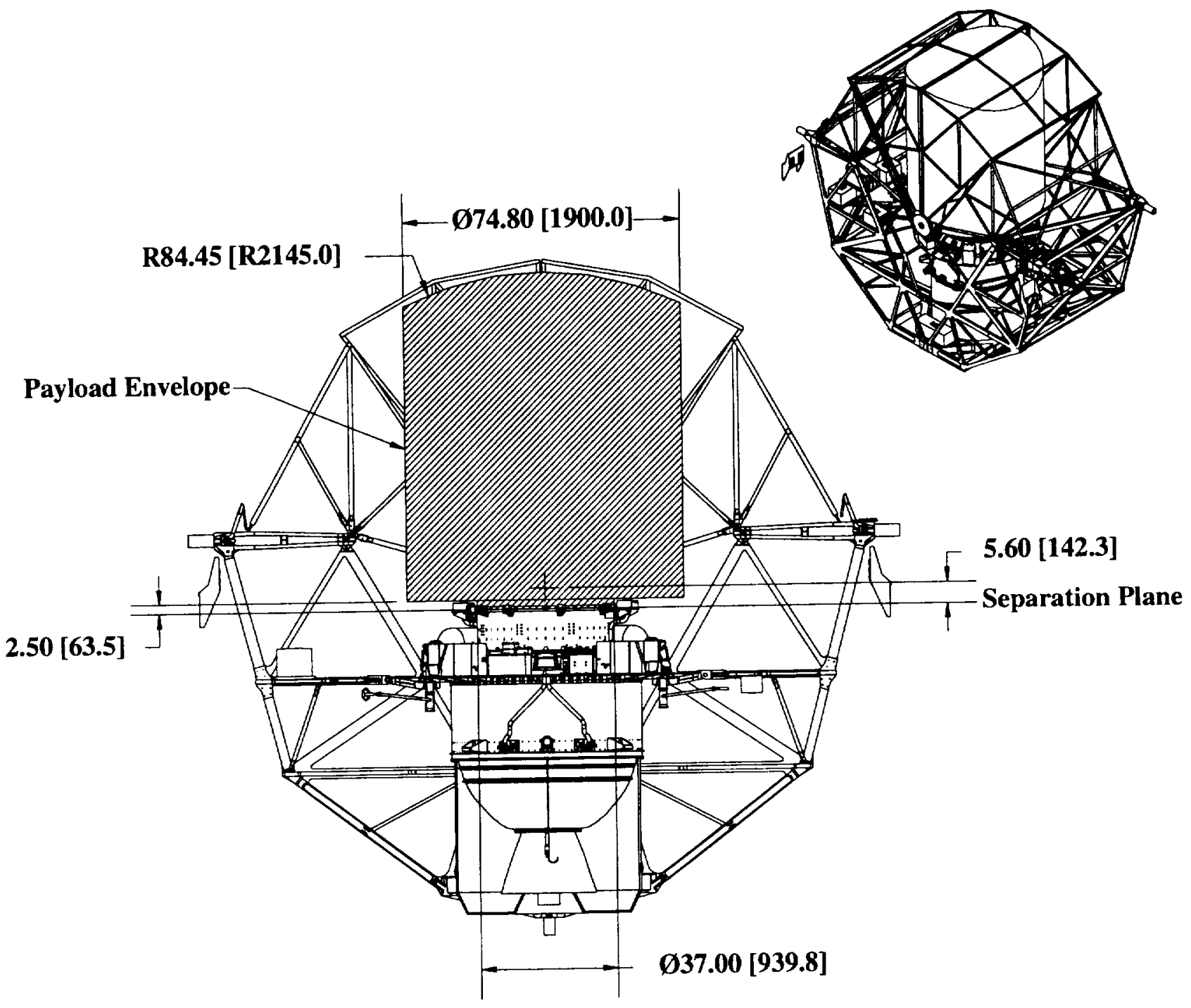

Figure 6 IRIS-GUS Physical Accommodation Envelope 